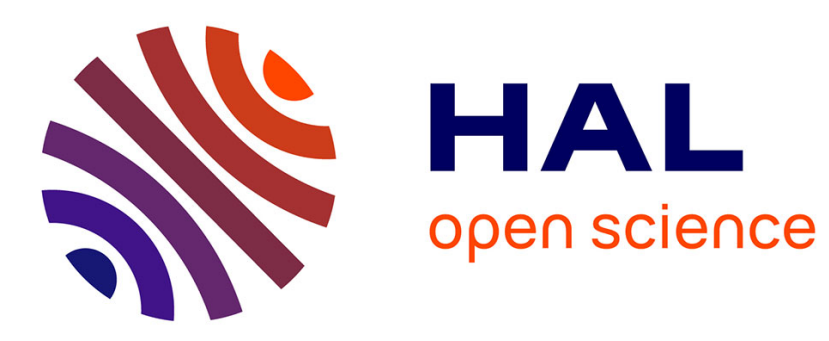

\title{
Kc and Gc at slow speeds for polymers
}

J.G. Williams

\section{To cite this version:}

J.G. Williams. Kc and Gc at slow speeds for polymers. D.R. Moore; A. Pavan; J.G. Williams. Fracture Mechanics Testing Methods for Polymers, Adhesives and Composites, 28, Elsevier, pp.11-26, 2001, European Structural Integrity Society, 978-0-08-043689-0. 10.1016/S1566-1369(01)80025-X . hal-03362289

\section{HAL Id: hal-03362289 \\ https://hal.science/hal-03362289}

Submitted on 1 Oct 2021

HAL is a multi-disciplinary open access archive for the deposit and dissemination of scientific research documents, whether they are published or not. The documents may come from teaching and research institutions in France or abroad, or from public or private research centers.
L'archive ouverte pluridisciplinaire HAL, est destinée au dépôt et à la diffusion de documents scientifiques de niveau recherche, publiés ou non, émanant des établissements d'enseignement et de recherche français ou étrangers, des laboratoires publics ou privés. 


\title{
$K_{\mathrm{C}}$ AND $\mathrm{G}_{\mathrm{C}}$ AT SLOW SPEEDS FOR POL YMERS
}

\author{
J.G. WILLIAMS
}

\section{INTRODUCTION}

A linear elastic fracture mechanics (LEFM) protocol for deternining $K_{c}$ and $G_{c}$ for plastics is reproduced as the appendix to this paper. This was the first protocol developed by TC4 and was chosen as a starting point because many members had experience of the test method and it was felt to be of practical importance. The basic method was that developed by ASTM for $\boldsymbol{K}_{\mathrm{c}}$ testing of metals [1] but with significant changes to make it suitable for polymers and to include $G_{c}$ determination. The version in the appendix is the final fonn produced by TC4 and was the basis used for the ASTM version [2] and subsequently the ISO version [3]. These latter contain changes made to conforn to the style and practices of those bodies, but none of substance occurred.

\section{BACKGROUND TO THE TECHNICAL ISSUES}

The major technical issues addressed in the protocol are notching and the definition of initiation. The method requires that a natural sharp crack is first grown and then the conditions for its re-initiation used to define $K_{r}$ and $G_{c}$. Great skill and care is required to produce these initial cracks and the results are critically dependent on their quality. Different techniques are required for different materials ranging from razor blade tapping in hard materials to blade sliding for soft materials. Initiation is defined as either the maximum load or the load which gives a $5 \%$ increase in compliance. Neither is true initiation but represents a reproducible value for a small amount of crack growth.

The size criteria for validity are designed to ensure both LEFM and plane strain and a further restriction, that the maximum load should be no more than $10 \%$ greater than that for the 5\% compliance change, is a guarantee on linearity and hence LEFM conditions. It is also worth noting that the energy result used to find $G_{\mathrm{c}}$ requires a compliance correction for load point indentation, a notion which arises in several protocols.

\section{RESULTS OBTAINED USING THE PROTOCOL}

An example of a set of data, in this case a nylon, is given below. Nine groups perforned the tests and it can be seen that the average standard deviation are 5\% for $K_{I C}$ and $12 \%$ for $G_{I C}$. The agreement between the two values of $E$ is generally good with differences of less than $1 \%$ for five sets of data and only one of up to $10 \%$. The data sets do show the common characteristic of such exercises in that some values are wildly out suggesting an error which is usually difficult to identify. Nylon is given as an example because it is not among those materials which are easy to notch (e.g. epoxies, PMMA), nor is it amongst those which are rather dif 
(e.g. PE, PP). However, with perseverance, good results can be achieved as demonstrated in Table 1.

TABLE 1. Results of the measurements of $K_{I C}$ and $G_{I C}$ performed on a Nylon by nine groups of ESIS TC4.

\begin{tabular}{|c|c|c|c|c|c|c|}
\hline Group No & $\begin{array}{l}\text { Specimen } \\
\text { type }\end{array}$ & Notching & $\begin{array}{l}K_{I C}(\text { mean }) \\
M P a . \sqrt{m}\end{array}$ & $\begin{array}{l}G_{I C} \text { (mean) } \\
\mathrm{kJ} / \mathrm{m}^{2}\end{array}$ & $\begin{array}{l}E_{\text {stiff }} \\
\mathrm{GPa}\end{array}$ & $\begin{array}{l}E_{\text {fract }} \\
\mathrm{GPa}\end{array}$ \\
\hline $\begin{array}{l}4 \\
5 \\
6 \\
7 \\
8 \\
9\end{array}$ & $\begin{array}{l}\text { SENB } \\
\text { SENB } \\
\text { SENB } \\
\text { SENB } \\
\text { SENB } \\
\text { CT } \\
\text { SENB } \\
\text { SENB } \\
\text { SENB }\end{array}$ & $\begin{array}{l}\text { RS } \\
\text { RT } \\
\text { RT } \\
\text { RS } \\
\text { RT } \\
\text { RT } \\
\text { RS } \\
\text { RT } \\
\text { RT } \\
\text { RT } \\
\text { RT }\end{array}$ & $\begin{array}{l}4.14 \pm 0.17 \\
4.03 \pm 0.10 \\
3.79 \pm 0.08 \\
3.84 \pm 0.17 \\
4.21 \pm 0.26 \\
4.10 \pm 0.35 \\
3.82 \pm 0.21 \\
4.46 \pm 0.13 \\
3.99 \pm 0.10 \\
3.9 \pm 0.3 \\
4.10 \pm 0.22\end{array}$ & $\begin{array}{l}4.76 \pm 0.98 \\
3.92 \pm 0.15 \\
4.01 \pm 0.17 \\
4.48 \pm 0.70 \\
4.82 \pm 0.73 \\
5.14 \pm 0.67 \\
4.20 \pm 0.41 \\
5.82 \pm 0.24 \\
4.80 \pm 0.46 \\
4.7 \pm 0.8 \\
6.40 \pm 0.8^{b}\end{array}$ & $\begin{array}{c}3.65 \\
4.14 \\
2.24^{\mathrm{a}} \\
3.32 \\
3.64 \\
3.30 \\
3.63 \\
3.57 \\
- \\
3.22 \\
-\end{array}$ & $\begin{array}{l}3.65 \\
4.14 \\
3.58 \\
3.33 \\
3.71 \\
3.28 \\
3.32 \\
3.42 \\
3.32 \\
3.21 \\
2.63^{b}\end{array}$ \\
\hline & & Mean & $4.03 \pm 0.19$ & $4.82 \pm 0.56$ & & \\
\hline $\begin{array}{ll}\text { a } & \text { Error } \\
\text { b } & \text { Witho } \\
\text { RS Razor } \\
\text { RT Razor }\end{array}$ & $\begin{array}{l}\text { ected } \\
\text { dentation } \\
\text { ling } \\
\text { ping }\end{array}$ & rrection & $\mathrm{E}_{\text {stiff }}=\psi$ & $B C, E_{f}$ & 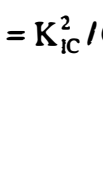 & \\
\hline
\end{tabular}

\section{CONCLUDING COMMENTS}

In general this protocol has worked well and has now been adopted as an ISO standard for plastics. It does incidentally work well for other materials, which are reasonably stiff and linear in their loading response, as would be expected. W th some additional procedures it has been used to measure toughness values in injection moulded discontinuous fibre composites [4] and more recently it has been successfully applied to foods [5].

\section{ACKNOWLEDGEMENTS}

The protocol was developed over about six years and many groups contributed results and insights, which gave rise to the final version. Below is a list of those contributors with their affiliation in 1990. 
Professor JG Williams: Dept of Mechanical Engineering, Imperial College, UK

Professor HH Kausch: Lab de Polymere, Dept de Materiaux, Ecole Polytechnique Federal de Lausanne, Switzerland.

Dr P Czarnocki: $\quad$ Warsaw University of Technology, Poland.

Dr G C Adams: $\quad$ EI Du Pont de Nemours \& Co Inc, USA

Professor W Bradley: Texas A \& M University, USA.

Dr MJ Cawood: $\quad$ BP Chemicals, UK

Dr ML Clerbois: $\quad$ Solvay et Compagnie, Belgium

Dr MH Daeniker, Du Pont de Nemours Int. SA, Switzerland

Dr P Davies: $\quad$ Universite de Compiegne, France

Dr GE Hale: $\quad$ The Welding Institute, Cambridge, UK

Dr Ing W Doll: $\quad$ Fraunhofer-Institute fur Werkstoff-mechanik, FRG.

Mr B Echalier: $\quad$ Atochem, France.

Mr M P Flueler: $\quad$ Swiss Federal Laboratories for Materials Testing (EMPA), Switzerland

Professor K Friedrich: Technische Universitat Hamburg, FRG

MrE Reese: $\quad$ Technische Universitat Hamburg, FRG

Mr H Wittich: $\quad$ Ciba-Geigy, AG, Switzerland

Dr KP Jud: $\quad$ Ciba-Geigy, AG, Switzerland

Mr M Fischer: $\quad$ Ciba-Geigy, AG, Switzerland

Professor AJ Kinloch: Imperial College, UK

Mr I Malkin: $\quad$ Instron Ltd, UK

Dr B Melve: $\quad$ Sintef, Norway.

Dr DR Moore: $\quad$ ICI Petrochemical \& Plastics Division, UK.

Professor A Pavan: $\quad$ Politecnico di Milano, Italy.

Dr F Ramsteiner, BASF, AG, FRG.

Dr A Roulin-Molony: Ecole Polytechnique Federal de Lausanne, Switzerland

Dr R Schirrer: $\quad$ EAHP, France

Dr SD Sjoerdsma: DSM, The Netherlands

Dr C Wrotecki, $\quad$ CdF Chimie SA, France

Dr Wutthrich: $\quad$ BBC, Baden-Datwil, Switzerland

\section{REFERENCES}

[1] ASTM E399-90: Standard Test Method for Plane-Strain Fracture Toughness of Metallic Materials, 1990.

[2] ASTM D5045-99: Standard Test Methods for Plane-Strain Fracture Toughness and Strain Energy Release Rate of Plastic Materials, 1999.

[3] ISO 13586-1: Standard Test Method for "Determination of Fracture Toughness $\left(\mathrm{G}_{\mathrm{C}}\right.$ and $\left.\mathrm{K}_{\mathrm{C}}\right)$-Linear Elastic Fracture Mechanics (LEFM) Approach." (2000).

[4] Moore, D.R., $K_{C}$ and $G_{C}$ at "Slow Speeds for Discontinuous Fibre Composites", in "Fracture Mechanics Testing Methods for Polymers, Adhesives and Composites" Elsevier Science, 2001.

[5] Kamyab, I., Chakrabarti, S. \& Williams, J.G. Cutting Cheese with Wire. Journal of Materials Science, 33, 2763-2770, 1998. 


\title{
7. THE TEST PROTOCOL
}

\author{
A Linear Elastic Fracture Mechanics (LEFM) Standard \\ for Deternining $K_{I C}$ and $G_{I C}$ for Plastics.
}

Testing Protocol - March 1990

This protocol has been created by the activities of the ESIS TC4 Task Group on Polymers and Composites and is the result of a series of Round-Robin tests. It is intended to form the basis of national and international standards. It has been drafted by Professor JG Williams, Mechanical Engineering Department, Imperial College London, UK.

\section{Introduction}

These tests are designed to characterise the toughness of plastics in terms of the critical stress intensity factor $K_{c}$, and the energy per unit area of crack $G_{c}$, at fracture initiation. The scheme used assumes liner elastic behaviour of the cracked sample, so certain restrictions on linearity of the load - displacement diagram and specimen width must be imposed to ensure validity. In addition, a state of plane strain at the crack tip is required so that thickness normal to the crack front must be sufficient to ensure this state. Finally the crack must be sufficiently sharp to ensure that a minimum value of toughness is obtained. These requirements are common to the ASTM metals standard E399 and much of what follows is drawn from this source. There are, however, special problems associated with plastics and these are accommodated in what follows. Items not covered here will be found in E399. It should also be noted that $G_{c}$ is of particular importance for plastics and this protocol covers its determination, while E399 does not.

\section{Specimen Preparation}

Three point bend (also called single edge notched bend, SENB) and compact tension (CT) geometries are recommended, because these have predominantly bending stress states which require smaller sizes to achieve plane strain than other configurations. It is usually helpful to maximise the thickness used from a sheet sample and this is best achieved by making the sheet thickness that of the specimen i.e. $B$ in Figure 1 where the two configurations are shown. In both tests the crack length range should be; $0.45<a / W<0.55$ and it is usually convenient to make $W=2 B$ initially.

\section{Notching}

The ideal case is when a natural crack is re-initiated and this is embodied in the metals test by requiring that an initial machined notched sample is fatigued to give some growth. This method may be used for plastics, but often it is difficult to do, because of unstable fatigue crack growth and the necessity of using low frequencies $(<4 \mathrm{~Hz}$ in some plastics) to avoid hysteretic heating. In plastics it is possible to produce 
sufficiently sharp initial cracks by other methods and particularly by first machining a sharp notch and then further sharpening it by using a razor blade. This is generally a
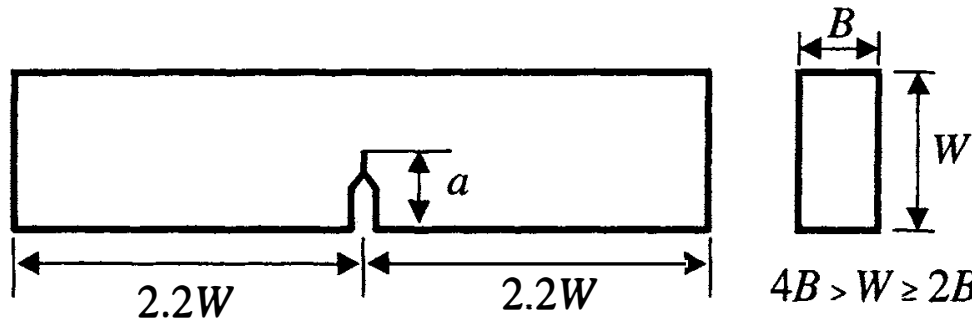

$4 B>W \geq 2 B$

a) Three point bend specimen (SENB)

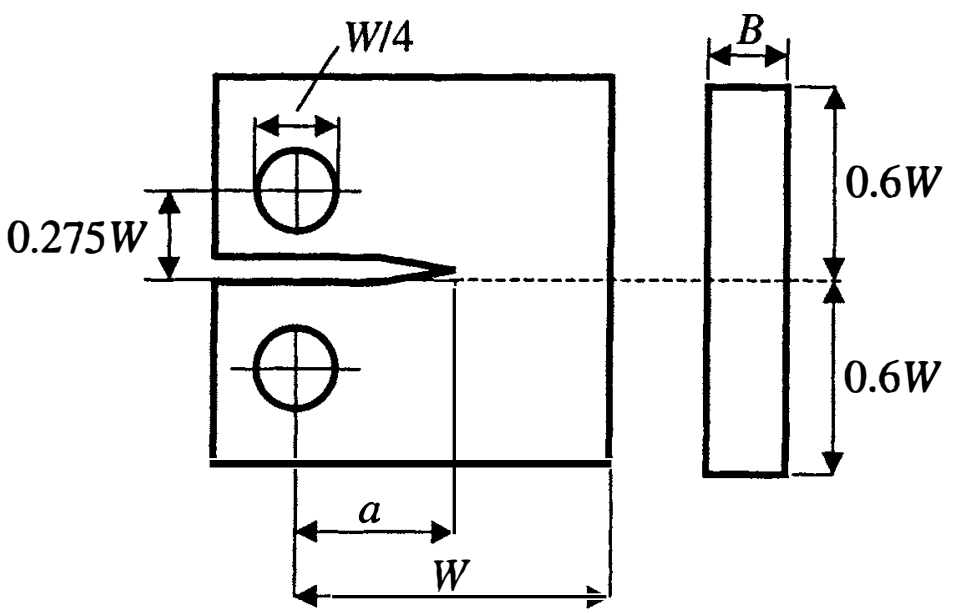

b) Compact tension configuration (CT)

Figure 1. Specimen configurations (as in document E399)

much simpler technique than growing cracks in fatigue. The procedure to be followed is:

i) Machine or saw a sharp notch in the specimen. Then generate a natural crack by tapping on a new razor blade placed in the notch. It is essential to persevere with this since in brittle specimens a natural crack can be generated by this process, but some skill is required in avoiding too long a crack or local damage. (Some precompression of the specimen may be helpful). The cracks grown should be several times longer than the pre-notch tip radius. Failure to generate a natural crack will result in too high values. 
ii) If a natural crack cannot be generated, as in some tough specimens, then the notch can be sharpened by sliding or sawing a new razor blade across the notch. Again the depth of this extension should be greater than the origilal notch tip radius. Pressing the blade into the notch is not recommended because of induced residual stresses.

\section{Test Conditions}

Sirsce plastics are viscoelastic materials it is necessary to specify both the temperature and time scale under which the result was obtained. As a basic test condition, it is recommended that $23^{\circ} \mathrm{C}$ and a crosshead rate of $10 \mathrm{~mm} / \mathrm{min}$ be used. In all cases the loading time should be quoted.

If it is not possible to obtain valid results at $23^{\circ} \mathrm{C}$ it is often possibie to do so by decreasing the temperature, which usually does not change $K_{r}$ greatly, but increases the yield stress rendering the fractures more brittle. If this procedure is used then again both temperature and loading time must be stated.

It is recommended that speeds of greater than $1 \mathrm{~m} / \mathrm{s}$ or loading times of less than lins should be avoided, because of the danger of dynamic effects causing errors.
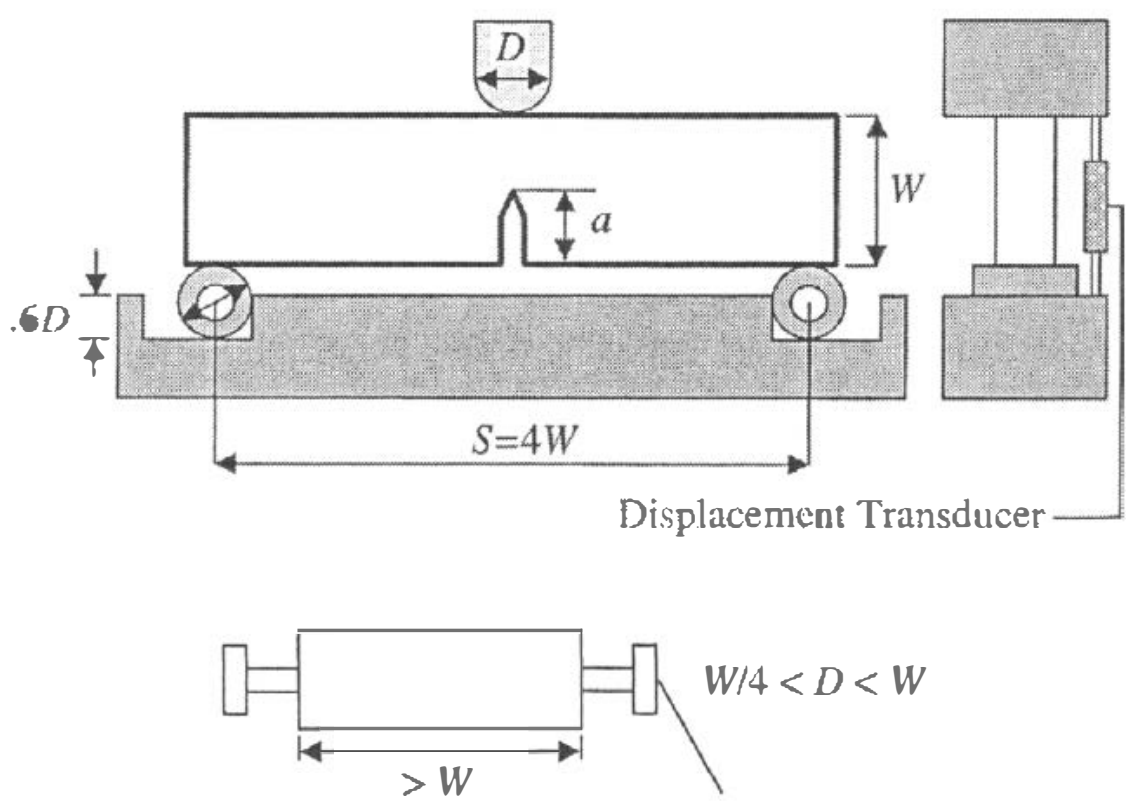

Bosses For Rubber Bands

(see method E399)

Figure 2. Bending rig used for SENB testing 


\section{Loading Rigs}

For SENB a rig with moving rollers ${ }^{1}$ of sufficiently large diameter to avoid plastic indentation is recommended. That shown in Figure 2 is based on E399. For the CT test the loading is via pins in the holes.

For either sample configurations, the displacement measurement can be performed using the loading machine's internally provided stroke (position) transducer. The fracture test displacement data must then be corrected for total system compliance, loading pin penetration and sample compression. This can be performed by a simple calibration of the testing system. The procedure is as follows.

A test configuration as shown in Figures $3 \mathrm{a}$ or $3 \mathrm{~d}$ using identically prepared, but unnotched, samples is used to generate a load-displacement correction curve. This correction curve is then "subtracted" from the load-displacement curve obtained during the actual fracture test with notched samples. This subtraction is performed by subtracting the correction curve displacement from the fracture test displacement at corresponding loads.

In practice, a linear correction curve can usually be obtained (up to the maximum loads recorded in the fracture test). Use of a linear correction simplifies the displacement correction. Any initial non-linearity due to penetration of the loading pins into the sample is observed during both the calibration test and the actual fracture test, so a linearisation of the near-zero correction data and the fracture test data can effectively correct for this initial non-linearity.

This displacement correction must be performed for each material and at each different temperature or rate. Polymers are generally temperature and rate sensitive and the degree of loading pin penetration and sample compression can vary with changes in these variables.

If the internally provided displacement transducer is not available, then externally applied displacement measuring devices may be used. For this case, displacement should be taken at the load point.

For CT samples, this is preferred to crack mouth opening since the load point displacement is required for the energy calculation used in finding $G_{c}$. For CT, a clip gauge near the pins will be satisfactory. (If a stiff metals gauge is used it may be necessary to correct the loads in a plastics test.)

For SENB a displacement transducer can be placed between the load point and the base as indicated in Figure 2. In the $G_{c}$ tests it is necessary to correct the measured displacement for indentation effects and machine compliance. This can be done by two methods:

\footnotetext{
${ }^{1}$ It has subsequently become more common to use fixed supports of the same radius as specified for the rollers. No significant errors have been observed by this simplification of the test rig.
} 
First method: A load displacement curve from that in Figure 3a may be subtracied from that in the fracture test to obtain the true displacement. The load-displacement curve is usually linear and its slope detennines the compliance due to indentation and machine stiffness, $C_{\text {cin }}$. The value here would be slightly high because of flexing, so a more precise result can be obtained from the second method described below.

Second method: Using the arrangement shown in Figure $3 \mathrm{c}$ the compliance of the machine, $C_{m}$, is deternined. This is subtracted from the compliance obtained from the arrangement in Figure $3 \mathrm{~b}$, to give the compliance due to the indentation at the centre point of the sample, $C_{i}$. Therefore, indention compliance due to both the loading striker and the rollers is given by $3 / 2 C_{i}$. Thus $C_{\text {rop }}$ is: $C_{m}+3 / 2 C_{i}$.

The indentation tests should be performed such that the loading times are the same as the fracture tests. Since the indentations are stiffer, this will involve lower rates to reach the same load; in many cases about half the speed. (More details on energy calculations are given in section 8 \%.

a)

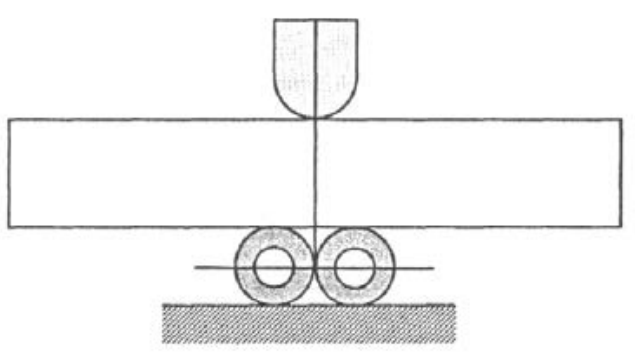

b)

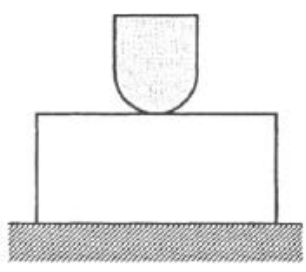

c)

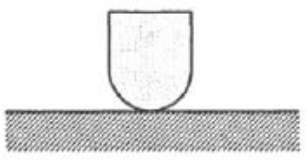

d)

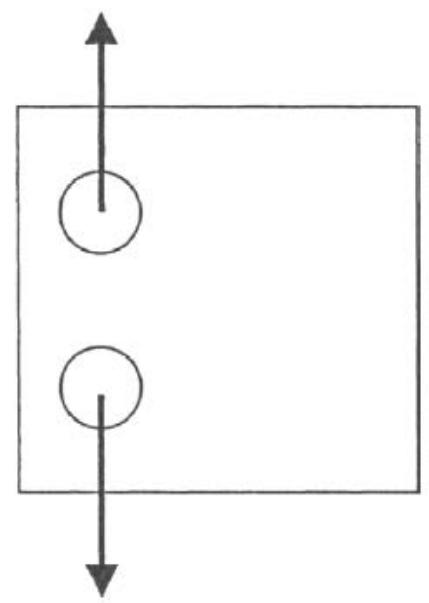

Figure 3. Arrangements for finding indentation displacement 


\section{Test Procedure}

It is recommended that three replicates be used. The test is performed and the load versus load-point displacement curve obtained. In the ideal case this is a linear diagram with an abrupt drop of load to zero at the instant of crack growth initiation. In some cases this occurs and $K_{Q}$ can be found from the maximum load. (In such cases a natural crack will be required, see section 3 ). In most cases there is some nonlinearity in the diagram and this can be due to plastic deformation at the crack tip, non-linear elasticity, general visco-elasticity and stable crack growth after initiation, but prior to instability. The first three effects violate the LEFM assumption and the fourth one means that the true initiation load is not defined by the maximum. Indeed it is doubtful if an exact definition of initiation could be made and with this, and a need for simplicity in mind, the arbitrary rule of E399 is used here. The diagram is shown (exaggerated) in Figure 4, and a best straight line is drawn to determine the initial compliance $C$ as shown. This is then increased by $5 \%$ and a further line drawn. If $P_{\max }$ falls within these two lines then $P_{\max }$ is used to find $K_{Q}$. If the $C+5 \%$ intersects the load curve then $P_{5 \%}$ is found and this is taken as the load at crack initiation. In fact if all the non-linearity is due to crack growth, then it corresponds to a particular amount of crack growth given by:

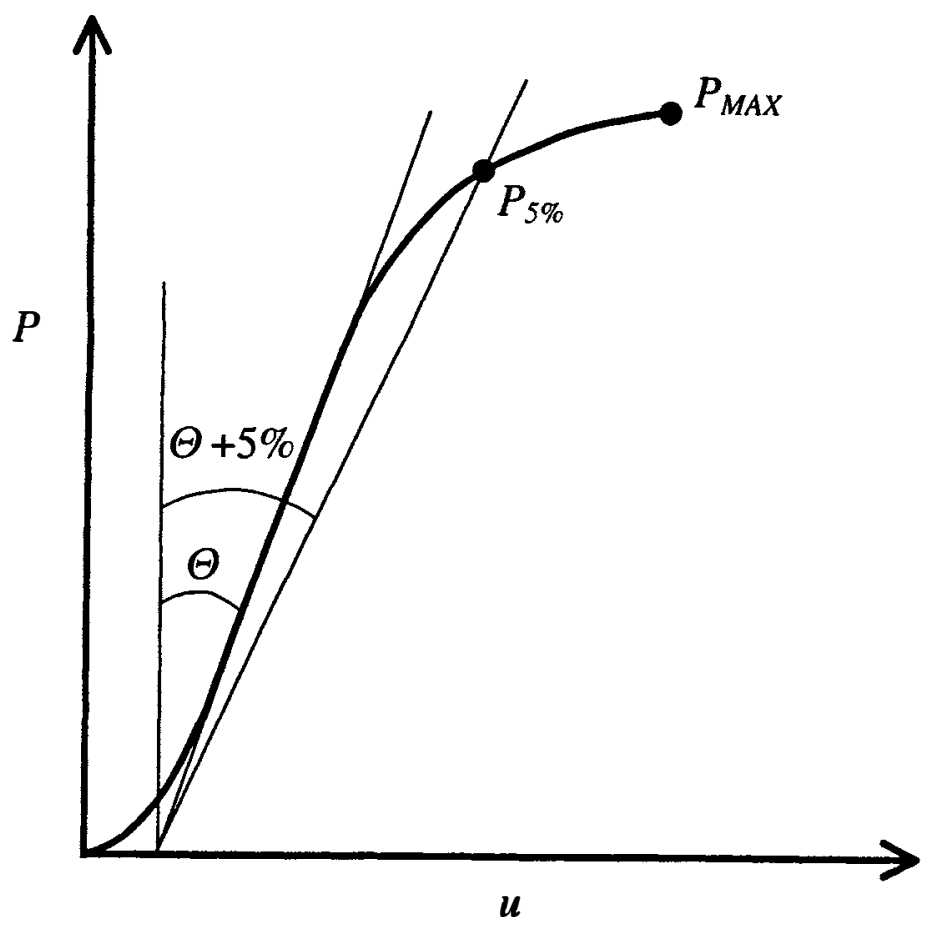

Figure 4. Determination of $P_{5 \%}$ and $C$ 


$$
\frac{\Delta a}{a}=\frac{\phi}{(a / W)} \cdot \frac{\Delta C}{C}
$$

Where $\phi(a / W)$ is the calibration factor discussed in section 8 . For the configuration in SENB used here, $\phi /(a / W) \approx 0.5$ so we have $\Delta a / a \approx 2.5 \%$ i.e. a $2.5 \%$ increase in crack length.

To stay within the LEFM condition it is further specified that:

$$
\frac{P_{\max }}{P_{5 \%}}<1.1
$$

i.e. a $10 \%$ non-linearity is allowed. If $P_{\max } / P_{5 \%}>1.1$ then the test is invalid. If $P_{\max } / P_{5 \%}<1.1$ then $P_{5 \%}$ is used in calculation of $K_{Q}$ or $P_{\max }$ it if falls within the two lines. (It should also be noted that crack 'pop-in' can occur in which the crack jumps forward a small distance and then arrests. This results in a short drop in the curve and then a continued rise. This value of load can be used and quoted as a 'pop-in' value.)

Values of $K_{Q}$ are computed from the original crack length $a$ which is bs:st determined from the fracture surface after testing. An average value may be used but the difference between the shortest and longest length should not exceed $10 \%$. Care should be taken that it is the original crack which is being observed since slow grow th can occur. $K_{Q}$ is then calculated from the following relationship:

$$
K_{Q}=f \frac{P_{Q}}{B W^{\frac{1}{2}}}
$$

Tabulated values of $f \phi, \psi$ and $\eta_{e}$ are presented in Table 1 for the SENB specimen and Table 2 for the CT specimen.

\section{Size Criteria}

The validity of $K_{Q}$ should now be checked via the size criteria;

$$
B, a,(W-a)>2.5\left(\frac{K_{Q}}{\sigma_{y}}\right)^{2}
$$

Since specimen dimensions require that $W=2 B$ initially (see section 2 ) and $a / W \approx 0.5$ then usually all are satisfied if one is. In fact the criteria covers two limitations in that $B$ must be sufficient to ensure plane strain but $(W-a)$ has to be sufficient to avoid excessive plasticity in the ligament. If $(W-a)$ is too small the test will usually violate the linearity criteria but not necessarily so. If the linearity 
criteria is violated a possible option is to increase $W$ for the same $B$. Values of $W / B$ of up to 4 are permitted. It should also be noted that if the specimen is too small $B$ will result in $K_{Q}$ being high whilst $(W-a)$ will result in it being low. The nett effect may be close to correct, but unfortunately in an unpredictable way, since the dependence on $B$ cannot be quantified.

$\sigma_{y}$ is the uniaxial tensile yield stress and for polymers this is conventionally taken at the maximum load. Because of visco-elastic effects the $0.2 \%$ offset value as used for metals is not a yield stress and gives too low a value. Shear yielding in tensile tests in most polymers can be achieved by carefully polishing the specimen edges, but if brittle fracture does occur then, since yielding is at a larger load, the stress at fracture may be used in the criteria to give a conservative size value. An alternative is to use 0.7 times the compressive yield stress. In all cases the time to yielding should be within $\pm 20 \%$ of the fracture loading time and the method of finding $\sigma_{y}$ given.

If these criteria are met then, $K_{Q}=K_{I C}$, the plane strain value.

\section{8. $G_{c}$ Calculations}

$G_{I C}$ can, in principle, be obtained from,

$$
G_{I C}=\frac{\left(1-v^{2}\right) K_{I C}^{2}}{E}, \text { (for plane strain) }
$$

but for plastics $E$ must be obtained at the same time and temperature conditions because of visco-elastic effects. Many uncertainties are introduced by this procedure and it is considered preferable to determine $G_{I C}$ directly from the energy derived from integrating the load versus load-point displacement diagram. The procedure to be followed is via $K_{Q}$ for validity testing and then to determine the energy $U_{Q}$ up to the same load point as used for $K_{Q}$, as shown in Figure 5a. The correction curve, as sketched in Figure 5b, is usually quite linear and the energy from indentation and machine compliance, $U_{c o r}$ can be estimated from $C_{c o r}$ from,

$$
U_{\text {cor }}=\frac{1}{2} P^{2} C_{c o r}
$$

where

$$
P=P_{5 \%} \text { or } P_{\max }
$$

The true fracture energy is,

$$
U=U_{Q}-U_{c o r}
$$

It is considered easier to correct for initial curvature by extrapolation as shown, but subtracting the total curves is permitted. Total energy corrections are usually $<20 \%$.

$G_{c}$ may be calculated from this energy $U$ via, $^{+}$ 


$$
G=\frac{U}{B W \phi} \text { or } \frac{\eta_{c} U}{B(W-a)} ; \quad \eta_{e}=\frac{1-a / W}{\phi}
$$

(The $\eta_{e}$ form is of the same form to that used for $\delta$ tests). Values of $\eta_{c}$ are gives in Table 1 for SENB and Table 2 for CT.

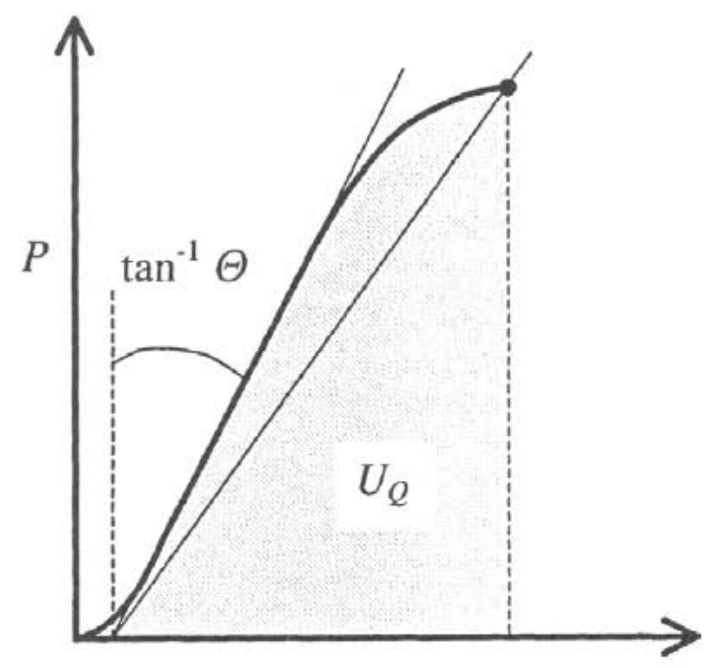

a) Load-deflection; Fracture test

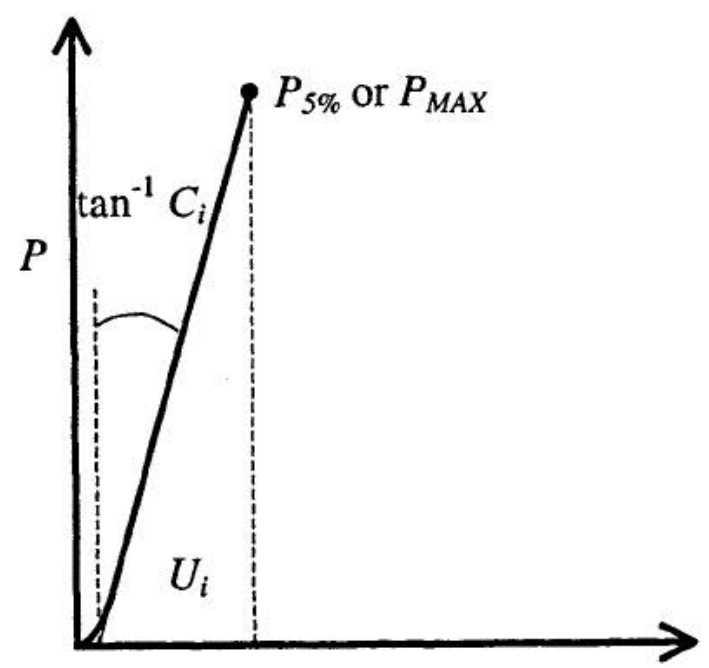

b) Load-deflection; Indentation

Figure 5. Method of correcting for indentation 
The energy calibration factor $\phi$ is defined as

$$
\phi=\frac{C}{\frac{d C}{d\left(\frac{a}{W}\right)}}
$$

and may be computed as shown in the Appendix . Values for the test geometries used are also given in Tables 1 and 2.

A useful cross check on accuracy may be made since $E /\left(1-v^{2}\right)$ can be found from the true compliance $C$, ie, $C=C_{Q}-C_{\text {cor }}$ from:

$$
\left(\frac{E}{1-v^{2}}\right) \cdot B C=2 f^{2} \phi=\psi
$$

and the factor $\psi$ is given in Tables 1 and 2. This value of $E /\left(1-v^{2}\right)$ should be compared with that obtained from $K_{I C}^{2} / G_{I C}$ and the former value should be the larger but the difference should be $<15 \%$. If the difference is greater, then the results should be examined for possible errors.

+ JG Williams, "Fracture Mechanics of Polymers", Ellis Horwood/Wiley, 1985.

\section{Reporting}

The following format for reporting results is suggested;

i) Specimen Configuration and Dimensions

ii) Notching method

iii) Temperature and Loading Rate

iv) One example of Load - Displacement diagram

v) $\quad P_{\max }$ and/or $P_{5 \%}$ values for all (3) specimens plus loading times

vi) $\quad P_{\max } / P_{5 \%}<$ or $>1.1$ ?

vii) $K_{Q}$ value

viii) $\sigma_{y}$ value at maximum load and loading time

ix) $2.5\left(K_{Q} / \sigma_{y}\right)^{2} ;<$ or $>B, W-a$ ?

$\mathrm{x}$ ) Energy value (indentation and machine compliance corrected?)

xi) $G_{I C}$ value via $\phi$ or $\eta_{e}$

xii) $\quad E /\left(1-v^{2}\right)$ via $C_{c}$

xiii) $E /\left(1-v^{2}\right)$ via $K_{I C}^{2} / G_{I C}$ 
Appendix: Calibration factors for SENB and CT specimens

\begin{tabular}{|c|c|c|c|c|}
\hline$a / W$ & $f$ & $\phi$ & $\psi$ & $\eta_{e}$ \\
\hline & & & & \\
\hline & & & & \\
0.05 & 2.50 & 1.502 & 18.7 & 0.63 \\
0.10 & 3.39 & 0.857 & 19.7 & 1.05 \\
0.15 & 4.07 & 0.641 & 21.2 & 1.33 \\
0.20 & 4.70 & 0.526 & 23.2 & 1.52 \\
0.25 & 5.36 & 0.449 & 25.7 & 1.67 \\
0.30 & 6.09 & 0.391 & 28.9 & 1.79 \\
0.35 & 6.93 & 0.345 & 33.1 & 1.89 \\
0.40 & 7.93 & 0.307 & 38.5 & 1.96 \\
0.45 & 9.14 & 0.275 & 45.9 & 2.00 \\
0.50 & 10.65 & 0.246 & 55.9 & 2.03 \\
0.55 & 12.57 & 0.220 & 69.6 & 2.04 \\
0.60 & 15.09 & 0.195 & 88.7 & 2.05 \\
0.65 & 18.51 & 0.170 & 116.4 & 2.06 \\
0.70 & 23.40 & 0.145 & 158.5 & 2.07 \\
0.75 & 30.84 & 0.120 & 228.2 & 2.08 \\
0.80 & 43.21 & 0.096 & 357.7 & 2.09 \\
0.85 & 66.76 & 0.072 & 643.7 & 2.08 \\
0.90 & 123.30 & 0.049 & 1484.6 & 2.05 \\
0.95 & 351.62 & 0.025 & 6148.3 & 2.01 \\
& & & & \\
\hline & & & & \\
\hline
\end{tabular}

Table 1 Calibration Factors for SENB geometry with $S / W=4$.

Note: Values calculated using:

Bakker, A. Compatible Compliance and Stress Intensity Expressions for the Standard three-point Bend Specimen. International Joumal of Fatigue and Fracture of Engineering Materials and Structures 13 (2), 145, 1990. 


\begin{tabular}{|c|c|c|c|c|}
\hline$a / W$ & $f$ & $\phi$ & $\psi$ & $\eta_{e}$ \\
& & & & \\
\hline & & & & \\
0.25 & 4.92 & 0.199 & 9.6 & 3.77 \\
0.30 & 5.62 & 0.208 & 13.2 & 3.36 \\
0.35 & 6.39 & 0.213 & 17.4 & 3.05 \\
0.40 & 7.28 & 0.213 & 22.5 & 2.82 \\
0.45 & 8.34 & 0.208 & 28.9 & 2.64 \\
0.50 & 9.66 & 0.199 & 37.1 & 2.51 \\
0.55 & 11.36 & 0.186 & 48.1 & 2.42 \\
0.60 & 13.65 & 0.170 & 63.6 & 2.35 \\
0.65 & 16.86 & 0.152 & 86.6 & 2.30 \\
0.70 & 21.55 & 0.133 & 123.2 & 2.26 \\
0.75 & 28.86 & 0.112 & 186.3 & 2.23 \\
& & & & \\
\hline
\end{tabular}

Table 2 Calibration factors for CT geometry

Note: Values calculated using:

J.A. Kapp, G.S. Leger and B. Gross;

Fracture Mechanics Sixteenth Symposium, ASTM, STP 868, pp 27-44.

Appendix

Compact Tension Specimen $(0.2<a / w<0.8)$

$f=\frac{(2+\alpha)}{(1-\alpha)^{\frac{3}{2}}}\left[0.886+4.64 \alpha-13.32 \alpha^{2}+14.72 \alpha^{3}-5.6 \alpha^{4}\right]$

$\phi=\frac{\left(1.9118+19.118 \alpha-2.5122 \alpha^{2}-23.226 \alpha^{3}+20.54 \alpha^{4}\right)(1-\alpha)}{\left(19.118-5.0244 \alpha-69.678 \alpha^{2}+82.16 \alpha^{3}\right)(1-\alpha)+2\left(1.9118+19.118 \alpha-2.5122 \alpha^{2}-23.226 \alpha^{3}+20.54 \alpha^{4}\right)}$

Where $\alpha=a / w$

Single Edge Notched Bend Specimen $\quad(0<a / w<1)$ 


$$
\begin{aligned}
& f=6 \alpha^{\frac{1}{2}} \frac{\left[1.99-\alpha(1-\alpha)\left(2.15-3.93 \alpha+2.7 \alpha^{2}\right)\right]}{(1+2 \alpha)(1-\alpha)^{\frac{3}{2}}} \\
& \phi=\frac{A+18.64}{d A / d \alpha} \\
& A=\frac{16 \alpha^{2}}{(1-\alpha)^{2}}\left[8.9-33.717 \alpha+79.616 \alpha^{2}-112.952 \alpha^{3}+84.815 \alpha^{4}-25.672 \alpha^{5}\right]
\end{aligned}
$$$$
d A / d \alpha=\frac{16 \alpha^{2}}{(1-\alpha)^{2}}\left[-33.717+159.232 \alpha-338.856 \alpha^{2}+339.26 \alpha^{3}-128.36 \alpha^{4}\right]+
$$$$
16\left[8.9-33.717 \alpha+79.616 \alpha^{2}-112.952 \alpha^{3}+84.815 \alpha^{4}-25.672 \alpha^{5}\right]\left[\frac{2 \alpha(1-\alpha)+2 \alpha^{i}}{(1-\alpha)^{3}}\right]
$$ 